\title{
Ciencias sociales, humanas y del comportamiento: dificultades regulatorias en países latinoamericanos y su impacto en la investigación en salud
}

\author{
Patricia Sorokin' \\ Universidad de Buenos Aires \\ María Angélica Sotomayor Saavedra ${ }^{2}$ \\ Universidad Santiago de Chile \\ Mirtha Andreau de Bennato ${ }^{3}$ \\ Universidad Nacional del Nordeste \\ Carmen Alicia Cardozo de Martínez ${ }^{4}$ \\ Universidad Nacional de Colombia \\ Marcela González ${ }^{5}$ \\ Escuela Latinoamericana de Bioética \\ Claude Vergès 6 \\ Universidad de Panamá \\ Luis Manuel López Dávila \\ Universidad de San Carlos de Guatemala \\ Laura Rueda Castro ${ }^{8}$ \\ Universidad de Chile
}

I Magíster en Bioética (Universidad de Chile-OPS/OMS), Magíster en Salud Pública y Doctora en Ciencias Sociales (Universidad de Buenos Aires, UBA). Docente- Investigadora (Facultad de Medicina, UBA) y miembro Comité de Ética (SAIC). Correo electrónico: patriciasorokin@hotmail.com

2 Abogada, Magister Derecho Seguridad Social (Universidad de Chile), docente Bioética (Universidad de Chile, Andrés Bello y Central), miembro CEI (Universidad de Santiago, SSMO, Clínica Las Condes y otros).

Correo electrónico: masotomay@gmail.com 


\title{
Resumen:
}

La investigación en ciencias sociales, humanas y del comportamiento debe tener un sistema especial de regulación y clara normatividad cuando esta se interrelaciona con investigaciones en las ciencias y disciplinas de la salud. Se exponen puntos coyunturales que evidencian la situación crítica de riesgos para la dignidad, imagen, reputación, credibilidad o inocencia del sujeto en estudio, grupos o comunidades; y propone que los formuladores de políticas públicas elaboren acciones específicas para el sector, orientadas a la toma de conciencia de los miembros de Comités de Ética, de los investigadores y de financiadores, en el respeto a los derechos de los participantes en la investigación, sean éstos individuos o comunidades.

Palabras clave: Investigación social, vulnerabilidad, regulación, riesgo, daño.

\section{Social, human and behavioral sciences: regulatory difficulties in Latin American countries and its impact on health research}

\begin{abstract}
:
Research dealing with social, human and behavioral sciences should have a special regulation and a clear normativity when it interrelates with research done in the health sciences and professions. Situational aspects that make it evident how risks to an individual's dignity, image, reputation, credibility or innocence are shown, a proposal is given to the people that formulate public policy for this specific sector, orienting them to consider a more consensus approach to the members of ethics committees, researchers and those who finance projects that might infringe the rights of the participants in the research projects, being these individuals or communities.
\end{abstract}

Key words: Social research, vulnerability, infringement, regulations, risk, harm.

3 Magíster en Bioética (Universidad de Chile- OPS/OMS). Doctora en Filosofía (Universidad Nacional del Nordeste, UNNE). Directora del Doctorado en Filosofía (UNNE). Correo electrónico: mirthabennato@hotmail.com

4 Odontóloga. MSc. Fogarty International Scholar en Ética en Investigación Biomédica y Sicosocial (OPS/OMS-CIEB), miembro del CIEB y profesora del Diploma en Investigación Clínica (Universidad de Chile).

Correo electrónico: carmen_aliciademartinez@yahoo.co.uk

5 Lic en Psicología (Universidad Favaloro). Especialista en Psico-oncología (UCES). Máster en Cuidados Paliativos (Universidad del Salvador-Pallium). Miembro Comité de Ética (CELABE). Correo electrónico: marcelagonzalez_psi@hotmail.com.ar

6 Médica, Máster en Bioética y Derecho (Universidad de Barcelona). Docente y Presidenta del Comité de Bioética (Universidad de Panamá); Miembro del Comité Internacional de Bioética (UNESCO); Vice-Presidenta REDBIOETICA/UNESCO. Correo electrónico: cverges2004@yahoo.es

7 Médico y Cirujano (Universidad de San Carlos de Guatemala, USAC), Máster en Bioética y Derecho (Universidad de Barcelona), Doctor en Salud Pública (INSP). Investigador Fac. CC.MM. CICS y Coordinador del Comité de Bioética en Investigación en Salud (USAC). Correo electrónico: luislopez@usac.edu.gt

8 Licenciada en Filosofía y Licenciada en Ciencias de la Ocupación, Terapeuta Ocupacional y Magister en Bioética (Universidad de Chile). Directora del Departamento de Terapia Ocupacional y Ciencias de la Ocupación (Universidad de Chile). Correo electrónico: Irueda@med.uchile.cl 


\section{Ciências Sociais, humanas e do comportamento: dificuldades regulatórias nos países latino-americanos e seu impacto na pesquisa em saúde}

\section{Resumo}

A pesquisa em ciências sociais, humanas e comportamentais deve ter um sistema especial de regulamentação e normatividade quando relacionada ao campo da saúde. No artigo são expostos pontos conjunturais que evidenciam a situação crítica de riscos para a dignidade, a imagem, a reputação, credibilidade ou inocência do sujeito ou grupo de estudo. Assim se propõe que os formuladores de políticas públicas promovam ações para favorecer a tomada de consciência dos membros de Comités de Ética, pesquisadores e financiadores, sobre a importância do respeito aos participantes da pesquisa.

Palavras chave: pesquisa social, vulnerabilidade, regulamentação, risco, daño.
"A pesar de que el valor social de la investigación sea una condición necesaria para ser éticamente aceptable, no es suficiente. Toda investigación debe desarrollarse de tal manera que incluya el respeto y la preocupación para con los derechos y el bienestar de las personas $y$ de las comunidades participantes.
Este respeto y preocupación es manifiesto en los requisitos para el consentimiento informado, asegurando que los riesgos están minimizados y son razonables a la luz de la importancia de la investigación, y otros requisitos discutidos en este documento.
Las investigaciones deben ser igualmente sensibles a los valores de justicia y equidad.
Esta preocupación es visible al elegir las personas cuyas necesidades de salud están investigadas, cómo se distribuirán los riesgos y beneficios anticipados y quién tendrá acceso al conocimiento y a las intervenciones". (CIOMS, 2016) ${ }^{9}$

\section{Antecedentes}

La investigación en el campo de las ciencias sociales ${ }^{9}$ recoge gran cantidad de datos que se vinculan con la esfera privada e íntima de las personas y por lo general, tiene por objeto interiorizarse de actitudes

$9 \mathrm{CIOMS} / \mathrm{WHO}$, International Ethical Guidelines for Health-related Research Involving Humans, 20 I6, [En línea]. [citado el I0 diciembre de 20 I 6] Disponible en: https:/cioms.ch/wp-content/uploads/20 I7/0 I MEB-CIOMS-EthicalGuidelines.pdf La traducción nos pertenece.

En este artículo nos referiremos a "investigación social" o del área social para distinguirla de la investigación en "ciencias biomédicas" o del área biomédica. 
individuales, de comportamientos grupales o de creencias, percepciones y representaciones sociales. Suele presentar un contacto muy estrecho entre investigador e investigado, trátese de un sujeto o una comunidad, lo que puede implicar de por sí una variable que puede facilitar, fortalecer o mediatizar la confianza entre ambos, variable que, a su vez, influye en la metodología a utilizar esto es, en el método científico.

En investigación "el bienestar de los informantes debe constituirse en la más alta prioridad y su dignidad, privacidad e intereses deben estar protegidos en todo momento y circunstancia"|0.

Por su parte, debido a la importancia que ha cobrado la investigación biomédica, genética y clínica para la terapéutica en general de las enfermedades asociadas a estas áreas, se ha sobrevalorado el trabajo en este campo frente al social, dando mayor peso a los efectos físicos, biológicos, bioquímicos o ambientales que pueda tener la investigación, sin ponderar los efectos deletéreos sobre la propia imagen, así como la ausencia de consideración moral sobre los valores personales o grupales. Sin embargo, se observa de manera creciente, el impulso hacia nuevos enfoques analítico-éticos de la investigación en todos los campos del conocimiento. En general, la investigación en el área biomédica busca evidencias físicas concretas de daño o lesión al paciente o a las comunidades, por lo que la categorización del daño se ha hecho con este énfasis, dejando de lado o considerando de menor rango, el riesgo cultural, afectivo y, en general, social de las personas y comunidades.

Tradicional e históricamente, la ética de la investigación en el campo de las ciencias biomédicas, ha tenido un desarrollo más intenso y sostenido que el manifestado en el campo de las ciencias sociales y humanas. Los experimentos biomédicos realizados con personas vulnerables y sin su voluntad, constatados en el denominado "Juicio a los Doctores" con posterioridad a la Segunda Guerra Mundial en Núremberg, Alemania, motivaron la formulación del Código de Núremberg sobre investigación científica en seres humanos. Este hecho marcó el hito inicial para el desarrollo de diversos instrumentos internacionales de protección ética y normativa en la investigación científica. Entre estos la Declaración de Helsinki de la Asociación Médica Mundial, cuya última versión data de $2013^{\prime \prime}$, y las Pautas Éticas Internacionales para la Investigación Biomédica Consejo de Organizaciones Internacionales de las Ciencias Médicas (ClOMS) ${ }^{12}$.

En los años 60 se producen ya diversas denuncias de notorio alcance por Maurice Pappworth (en su libro "Human Guinea Pigs", 1967) y por Henry K. Beecher (en su artículo Ethics and clinical research, publicado en el NEJM, 1966), pero es a comienzos de los años setenta cuando alcanza notoriedad la publicación de los dos casos que cambiarían el curso de la historia: el estudio de la

I0 UNESCO. Ethical Guidelines for International Comparative Social Science Research in the framework of MOST, Artículo I0. 20 16. [En línea] [citado el 10 diciembre de 2016]. Disponible en: http://www.unesco.org/most/ethical.htm

II Asociación Médica Mundial (AMM). Declaración de Helsinki, Principios éticos para las investigaciones médicas en seres humanos, 64 Asamblea General, Fortaleza, Brasil, 20 I3. [En línea] [citado el 10 diciembre de 20 I6]. Disponible en: http://www.wma.net/ es/30publications/IOpolicies/b3/

$12 \mathrm{ClOM} / O M S$. Pautas éticas internacionales para la investigación biomédica en seres humanos. Ginebra 2002. [En línea] [citado el 10 diciembre de 2016$]$. Disponible en: http://www.cioms.ch/publications/guidelines/pautas_eticas_internacionales.htm 
sífilis en Tuskegee (una pequeña ciudad del Condado de Macon, en el Estado sureño de Alabama) y los estudios de hepatitis de Willowbrook (una escuela estatal del Estado de Nueva York). Desde entonces han quedado ligados a la idea de prácticas no éticas de investigación con seres humanos ${ }^{13}$.

Las violaciones señaladas, muestran la necesidad de establecer instancias de revisión experta y con formación en Ética. Surgen así los Comités de Investigación Científica y sus regulaciones correspondientes, dirigidas a la revisión de "protocolos biomédicos por investigadores biomédicos, con el propósito de proteger a los sujetos de los riesgos físicos de la creciente experimentación farmacológica y quirúrgica"'l4.

Así entonces, de la evidencia de los daños y su funesto legado, ha obtenido su identidad todo el sistema de protección en la investigación humana. No obstante, este énfasis se ha centrado en la integridad física y en el funcionamiento del organismo humano y así lo demuestra el desarrollo normativo ético internacional, que no considera la posibilidad de daño en otros aspectos fundamentales de la vida humana: respecto a la integridad psíquica, al entorno social humano y a la dimensión social de éste, que es el campo de estudio propio de las ciencias sociales.

Tampoco se consideran aspectos específicos de las percepciones, creencias, convicciones y conocimientos socio-culturales, que son vitales para las personas, pero lejanos para el investigador. Se estima que el respeto está asociado a la inviolabilidad del cuerpo y de los aspectos físicos, mas no de su esencia y sentido de vida. Esta situación pone en evidencia la multiplicidad y la complejidad de los aspectos antropológicos, culturales y psicológicos implicados, siendo éstos de difícil codificación por no resultar tan visibles como los datos físicos y biológicos; además no son de interés para el desarrollo de las eventuales "moléculas" que permiten el registro de la patente y consecuentemente el rédito económico.

Tales dimensiones de lo humano, claramente susceptibles de ser dañadas en una investigación científica, implican que el riesgo debe ser ampliamente conocido y visibilizado por el investigador, los financiadores y la misma comunidad para, asimismo, evitarlo o disminuirlo al máximo. Los aspectos señalados son motivo de la profunda deliberación que deben realizar los Comités de Ética de la Investigación donde, al igual que en la investigación biomédica, se requiere contar con elementos de carácter normativo, que formulen exigencias éticas, con la misma fuerza coercitiva.

\section{Investigación en el área de las ciencias humanas y sociales}

Las estrategias metodológicas que usualmente se aplican en esta área, implican una respuesta verbal o escrita que supone una participación voluntaria de los sujetos de investigación. Pero, el

13 de Abajo Iglesias, F. Un caso histórico: los estudios de hepatitis de la escuela estatal de Willowbrook Investigación pediátrica clínica y traslacional en la era genómica. S/I, 20 I2. [En línea] [citado el I 0 de diciembre de 20 I 6]. Disponible en: http://contenidos. institutoroche.es/pdf/20 I/libro_pediatria/anexos_capt4.pdf

14 UNESCO. Op. Cit. 
debido respeto a la privacidad de los sujetos de investigación está frecuentemente soslayado, aun en estudios en los que se indaga en cuestiones de índole altamente sensible; por ejemplo, cuando se trabaja con grupos focales o cuando se reportan testimonios o imágenes sin haber obtenido consentimiento previo.

La significación social que poseen los estudios relativos a la salud, tanto física como mental adquiere vital relevancia habida cuenta del caudal de información obtenida y circulante. Ésta, de no ser protegida ni resguardada, bien podría potenciar la impronta de los estigmas hacia personas, familias, grupos étnicos, religiosos, sociales y/o sub-culturales si el secreto (entendido éste como la preservación y el resguardo de la información confidencial que una persona le transmite a otra confiando en que ésta será mantenida en el orden de lo privado) se revelara, causando así daños y riesgos no previstos al momento de diseñar y/o implementar un estudio.

Por otra parte, la superposición de investigaciones de carácter clínico o biomédico (ensayos clínicos internacionales de la industria farmacéutica) con investigación social, implica que, como exigencia de la primera, el investigador deba aplicar las normas establecidas en las denominadas "Buenas Prácticas Clínicas" de la Conferencia Internacional de Armonización ${ }^{15}$ en las cuales no se consideran particularidades sociales, por estar concebidas para investigación del área farmacológica.

\section{Derechos de los sujetos de investigación}

La dignidad del ser humano como ser único, inalienable y merecedor de consideración y respeto, está en la base de los demás derechos y pese a ello es la que está en mayor riesgo de ser vulnerada en la investigación científica. Resulta evidente que la autonomía o autodeterminación que es parte del reconocimiento de esa dignidad, está radicada en su dimensión trascendente o relacional intersubjetiva, esto es situada más allá de las condiciones biológicas del ser humano expresadas en valores.

La protección de la esfera íntima del sujeto, el resguardo de su vida privada y la de su familia, como manifestación del respeto a la dignidad del ser humano, son derechos reconocidos y relevados en la Declaración Universal de Derechos Humanos, al disponer que "Nadie será objeto de injerencias arbitrarias en su vida privada, su familia, su domicilio o su correspondencia, ni de ataques a su honra o a su reputación. Toda persona tiene derecho a la protección de la ley contra tales injerencias o ataques"16. Dicha norma se reitera en el Pacto Internacional de los Derechos Civiles y Políticos ${ }^{17}$.

15 Conferencia Internacional de Armonización (ICH) Normas de Buenas Prácticas Clínicas. (BPC) 1997- I998. [En línea] [citado el 10 diciembre de 2016]. Disponible en: http://www.fda.gov/downloads/Drugs/../Guidances/ucm073 I 28.pdf

16 Naciones Unidas, Declaración Universal de Derechos Humanos, Adoptada por la Asamblea General de las Naciones Unidas en su Resolución 217 A (III), el 10 de diciembre de 1948 en París, Francia. [En línea]. [citado el 10 diciembre de 20 I6] Disponible en: http://www.un.org/es/documents/udhrl

17 Naciones Unidas, Pacto Internacional de los Derechos Civiles y Políticos, 1966, art. 17. [En línea] [citado el 10 diciembre de 2016]. Disponible en: http://www.ohchr.org/SP/Professionallnterest/Pages/CCPR.aspx 
Por su parte, el derecho a investigar es parte del derecho a la libertad creadora, consagrado como derecho económico, social y cultural, en el Pacto del mismo nombre, de Naciones Unidas $1966^{18}$.

Sin embargo, el ejercicio a la libertad creadora y a la libertad para investigar tiene sus límites en el obligado respeto a los derechos humanos. La dignidad del ser humano podría ser vulnerada mediante observaciones, cuestionarios con preguntas abiertas o cerradas, entrevistas, seguimiento de historias clínicas, y también, foros de discusión por Internet entre poblaciones en condición de vulnerabilidad y profesionales de la salud, entre otras intervenciones. Debe considerarse, asimismo, que como consecuencia de tales intervenciones, existe el riesgo de pérdida de la confidencialidad si el investigador, su equipo y todos aquellos que tengan o puedan tener acceso a la información sensible, la circularan irrestrictamente o sin control.

La libertad para investigar, relevada en su importancia, además de que puede atentar a derechos inalienables del sujeto, puede producirle daño, dolor o menoscabo. En investigaciones del área social con cierta frecuencia las entrevistas en profundidad, las encuestas o los estudios observacionales, pueden constituir claras intromisiones en la esfera privada o íntima de las personas que, aun habiendo otorgado su consentimiento, pueden no haber visualizado su riesgo personal o colectivo, como pudiere ocurrir a modo de ejemplo, al actualizar recuerdos o experiencias dolorosas. Ello se debe también a la falsa percepción social sobre la ausencia de riesgos y por ende a la falta de previsión sobre la necesidad de reparación de los daños o efectos secundarios de la investigación.

Existen otros daños, tales como "daño a la persona", "daño a la intimidad", "daño al proyecto de vida", "daño a la vida de relación", "daño a la autoimagen" que no son reparables mediante una compensación económica y en esta categoría también incluimos aquellos casos en los cuales afectándose la dignidad de las personas se produce una "pérdida de oportunidades", a través de la utilización de su imagen o exponiendo públicamente su historia clínica y/o sus datos personales ("toda información referida a personas físicas y de existencia ideal determinadas o determinables") y sus datos sensibles ("origen racial y étnico, opiniones políticas, convicciones religiosas, filosóficas o morales, afiliación sindical como a la información referente a la salud o a la vida sexual" Cfr. Ley argentina $N^{\circ} 25.326$ ).

Se vislumbra así que algunos sujetos pueden presentar debilidad frente a la investigación como los pobres, las comunidades rurales y los habitantes de pueblos originarios. La vulnerabilidad que afecta a una parte importante de la población mundial, incluida Latinoamérica, apela a una Ética de la Fragilidad, cuyo objeto es la protección del desamparado también en investigación, siendo "violencia toda intromisión en la vida privada de las personas, sin su consentimiento, toda coacción o manipulación de sus ideales de vida"|l.

La necesidad del Estado de disponer de información privada de los individuos, por ejemplo, por razones de salud pública y bien común, es incuestionable, sin embargo, no se puede ni se debería

I8 Naciones Unidas, Pacto Internacional de los derechos Económicos, Sociales y Culturales, 1966. [En línea] [citado el 10 diciembre de 2016]. Disponible en: http://www.ohchr.org/SP/Professionallnterest/Pages/CESCR.aspx

19 Gracia, D. Bioética Clínica, Ed. El Búho, Bogotá, 1998, págs. 39. 
abusar de esta prerrogativa y tampoco se puede trasladar este derecho a la investigación individual o de grupos particulares.

\section{Según Diego Gracia:}

"junto a la economía y la política, la racionalidad estratégica ha invadido un tercer campo, el derecho. El derecho propio de la razón instrumental o estratégica es el llamado positivismo jurídico, con sus cuatro notas de positividad (el derecho no se funda más que en la voluntad de legislador soberano, quien promulgándolo regula convencionalmente situaciones sociales), legalidad (no hay normas o criterios éticos anteriores al derecho, sino que este surge como modo de resolver conflictos de intereses), formalismo (es justo todo lo que cumple los requisitos formales o procedimentales establecidos por la ley, e injusto lo contrario) y, finalmente, utilitarismo (lo que el derecho busca es proteger los intereses de todos los implicados, o al menos de la mayoría"20.

Frente a la necesidad de hacer investigación, incluso en áreas que manejan "big data" sanitario, se plantea la necesidad de trabajar sobre la base de información previamente anonimizada o codificada, a efectos de garantizar la confidencialidad y de promover medidas de seguridad informática que eviten potenciales "ciberataques" con fines completamente distintos de aquellos para los cuales fue colectada.

\section{Consentimiento informado}

Como la manifestación más clara del respeto a la autonomía personal como parte del resguardo de la dignidad humana, debe garantizarse la expresión explícita de aceptación de participar en una investigación, acto o momento investigativo, debe respetarse por tanto el consentimiento, que debe ser previo, expreso, libre esto es exento de presión, e informado, del sujeto de investigación. El consentimiento es un proceso deliberativo verbal o escrito, que implica un encuentro dialógico que puede implicar varios momentos y concluir con un documento escrito que da fe del proceso. Es indispensable la información comprensible para el sujeto, que lo lleva a consentir sobre la base de la existencia previa de una comunicación auténtica, es decir transparente y bidireccional.

Es sabido que en algunas culturas se desconfía de lo escrito y así en estudios del área social suele ser un problema ético el "cómo" acreditar el carácter voluntario de la participación de los sujetos en la investigación. Cabe reconocer también que hay situaciones en las cuales, por el tipo de investigación, el hecho de informar a los sujetos de algún aspecto de la misma, puede comprometer la validez de ésta ${ }^{2 !}$. Si bien esos casos pueden ser excepcionales, como se señaló, el Informe Belmont

20 Gracia D. Profesión médica, investigación y justicia sanitaria, Ed. El Búho, Bogotá, 1998, pág. 67.

21 Comisión Nacional para la protección de sujetos humanos en investigación biomédica y comportamental. El Informe Belmont. U.S.A. 1979. [En línea] [citado el 10 diciembre de 2016]. Disponible en: http://www.bioeticayderecho.ub.edu/archivos/norm/ InformeBelmont.pdf 
específica que "sólo están justificados si queda claro que:" y establece tres excepciones, la primera si "la información incompleta es verdaderamente necesaria para lograr las metas de la investigación", la segunda es si "no se deja de revelar a los sujetos los riesgos que sobrepasen el mínimo" y la tercera si "hay un plan adecuado para informar a los sujetos, cuando sea apropiado, y para informarles los resultados de la investigación" 22 .

De todos modos, no se debe reservar "información acerca de los riesgos con el propósito de obtener la cooperación de los sujetos y siempre deben darse respuestas veraces a las preguntas directas acerca de la investigación. Hay que ser cuidadosos en distinguir los casos en que la revelación destruiría o invalidaría la investigación de aquellos en que, simplemente, sería un inconveniente para el investigador"23.

Son conocidos también los riesgos en algunas investigaciones en las cuales, según los investigadores, la participación voluntaria no es "deseable" pues conduce a sesgos o conductas desorientadoras para la investigación por lo que se recurre, y aun acepta, al engaño ${ }^{24}$. El abuso de esta opción fuera de la imperiosa necesidad de la ciencia, podría llevar a considerar que quienes investigan con participantes en tales estudios, no reconozcan u olviden el complemento de la autonomía definida en el pensamiento kantiano de la ética del deber, que señala el respeto que merecen los agentes autónomos y el derecho a ser protegidos cuando presentan condiciones o situaciones de autonomía disminuida. Se produce así un choque de apreciaciones entre los cultores de la disciplina y quienes evalúan lo ético, con la consiguiente violación del segundo imperativo Kantiano que prescribe "Obra de tal modo que uses a la humanidad, tanto en tu persona como en la persona de cualquier otro, siempre al mismo tiempo como fin y nunca simplemente como medio"25.

\section{Falencia regulatoria en la Investigación en Ciencias Sociales}

La investigación científica en el área de las ciencias humanas y sociales presenta escasez normativa específica, lo que deviene necesariamente en procesos de ajustamiento de las normas originalmente concebidas para las ciencias biomédicas, a una realidad distinta de aquella para las cuales éstas fueron concebidas, lo que provoca una tensión, tanto en los Comités como en los investigadores del área social, que enfrentan un nuevo desafío para el cual tampoco están preparados. Ello además del inconveniente que implica que, en este ejercicio, se debilite la protección de algunos derechos de las personas.

La falencia regulatoria en la materia, ha sido enfrentada sin que se haya logrado, hasta la fecha, consensos éticos a nivel local, regional ni global. La necesidad de regulación específica es evidente. Aún más, la evidencia muestra que cada vez más se incorpora la investigación social en el campo de

\footnotetext{
22 Ídem.

23 Idem.

24 Milgram, S., Obedience to Authority; An Experimental View. Harpercollins (ISBN 006 I 3 | 983X). Editor London: Harper Collins. 1974.

25 Kant, I. Cimentación para la metafísica de las costumbres. C. M. Ramírez (trad.) Buenos Aires, Aguilar, 5a . Ed. 1978 , págs. I I I - I 12.
} 
la salud ${ }^{26}$ y que, para la publicación de resultados, las revistas de arbitraje internacional requieren la revisión previa de Comités de Ética de la Investigación. No estando definidos los parámetros o criterios de evaluación, los Comités adaptan los existentes para la investigación experimental biomédica, lo cual claramente puede generar resultados débiles o confusos.

\section{Comités de Ética de la Investigación}

En la actualidad los Comités son una exigencia en centros de investigación y universidades, entre otros. Sin embargo, se debiera evitar la existencia de tales Comités y la integración de sus miembros como una obligación, con miras sólo al cumplimiento de normativas, que eventualmente se desconocen y a aprobar los proyectos de tales instituciones para no perder becas o subsidios, bajo un claro conflicto de interés. Esto dista significativamente de su rol ideal, que es la defensa del bienestar y de los derechos del sujeto de investigación; y, se asemeja al muy cuestionable "IRB shopping" (práctica mediante la cual los patrocinadores de ensayos clínicos eligen a quien remitir su propuesta, basándose en la suposición de que el Comité escogido aprobará su protocolo rápidamente y sin objeciones).

Los Comités debieran estar registrados, acreditados y evaluados en sus actividades por una autoridad central con poder de contralor, y la presencia de profesionales de las ciencias sociales en los Comités de Ética de la Investigación y en los del área social, debiera ser absolutamente necesaria.

En relación con la situación normativa percibida, los Comités de Ética de Investigación del área biomédica, especialmente aquellos que se hallan insertos en instituciones de educación superior, perciben incomodidad y eventualmente cierta incompetencia para hacer la revisión ética de los proyectos en los cuales hay investigación en colectivos o comunidades, puesto que, aplicadas las normas del área referida, inclusive no clasificarían como proyectos de riesgo. Aún más, algunos Comités no reciben tales estudios y otros se consideran compelidos a forzar la adaptación ad hoc de criterios y de procedimientos que fueron desarrollados para su aplicación en el campo de la investigación clínica o epidemiológica. Por su parte, los investigadores pueden sentirse violentados a cumplir requisitos y a adaptarse al cumplimiento de normas no específicas para tal campo de estudio y cuya implementación pudiere desfigurar el estudio original.

Claramente, la investigación social, carece de parámetros claros que vinculen en su desarrollo al investigador con obligaciones no solo éticas fundamentales sino jurídicas. Por otra parte, puede no ser fácil distinguir y definir en la práctica cotidiana, la información personal que puede dañar a una persona, de aquella que es imprescindible para los resultados de la investigación y su posterior aplicación. Por esto se requiere la mayor definición tanto conceptual como normativa, cuya deficiencia se visualiza. No se trata entonces, sólo de definir en una norma qué es de un ámbito o de otro o qué aspectos pondrán en riesgo a las personas, a las familias, a las comunidades y/o a las regiones geográficas.

26 Ulin P., Robinson E. y Tolley E. Investigación aplicada en salud pública. Métodos cualitativos. OPS / OMS, Washington D.C. 2006, 286 págs. [Publicación Científica y Técnica No. 614] 
En este sentido, por tradición también heredada del campo biomédico, la investigación social con altos estándares y credibilidad, es la realizada en grupos cerrados o en comunidades en la cuales se reconoce actualmente el mayor riesgo de lesión social o personal, dadas precisamente las condiciones en las que aquellas viven, interactúan o basan sus creencias. Así, en una revisión de tesis de estudiantes de Psicología, los autores aprecian la ausencia de observancia en "los usos semánticos, al utilizar en títulos y textos vocablos que promueven un menoscabo de la imagen de los participantes, con el consiguiente riesgo de estigmatización. Paradigma de tal cuestión es la referencia a 'drogadictos' o ‘esquizofrénicos”’27.

Por otra parte, la necesidad de comprensión de la importancia de los factores sociales para el control de las enfermedades crónicas, ha traído consigo el requerimiento de valorar los factores determinantes de la salud, pues se reconoce en ellos el verdadero eje sobre el cual se están produciendo los conflictos y sobre el cual asimismo, habría que realizar acciones para corregirlos, atenuarlos o eliminarlos, con la misma comunidad y a partir de sus propios conocimientos.

\section{Otras situaciones vinculadas}

En la hipótesis de un paraíso artificial del neuro-mejoramiento, se afectaría nuestra experiencia de la vida, el mundo vivido, en cuanto al sentido de la realidad, de la identidad y la libertad de la condición humana ${ }^{28}$.

Algo más cotidiano, como una investigación biomédica sobre un nuevo medicamento podría levantar esperanzas en cierta persona y depresión o pérdida de la fe, si sus expectativas se vieran frustradas. Ello implica que la investigación biomédica tiene efectos que van más allá de los clínicos y del producto en estudio. Un resultado positivo del denominado "efecto placebo" identificado por Henry K. Beecher ${ }^{29}$ en la década de los cincuenta y que corresponde al "conjunto de efectos sobre la salud que produce la administración de un placebo, que puede ser en forma de pastilla, una terapia, la automedicación o un mero acto médico, y que no se deben al efecto específico de ningún acto médico ${ }^{30}$. Se produce en sujetos que, recibiendo un sustituto del elemento en estudio, de apariencia similar, pero sin la droga activa, presenta efectos clínicos terapéuticos que bordean el 30\%.

27 Winkler, M. I.; Alvear, K.; Olivares, B. y Pasmanik, D. Querer No Basta: Deberes Éticos en la Práctica, Formación e Investigación en Psicología Comunitaria. Psykhe. 2012, vol.21, n. I, pp.I I5-129. [En línea] [citado el 10 diciembre de 20I6]. Disponible en: http://dx.doi.org/l 0.4067/S07/8-222820/2000 I00008.

28 Cf. Mainetti, J. A. Bioética y Medicina Poshumanística. Editorial Quirón, La Plata. 2013, págs. 60.

29 Cf. Beecher, H. K. The Powerful Placebo. 1955. Journal of the American Medical Association I59 (I7): I602-I606. ISSN 00029955. doi: I 0. 100 I/jama. 1955.02960340022006.

30 Para profundizar en las implicancias del uso de placebo y su relación con estudios sobre depresión, a ser evaluados por un Comité de Ética de la Investigación, véase Sorokin, P.; Valerio, C. y Cecchetto, S. "Poblaciones vulnerables e investigación biomédica: a propósito de un caso" en S. Bergel (coord.) Número especial de Bioética y Derechos Humanos de la Revista Jurídica de Buenos Aires. UBA-LexisNexis-Abeledo Perrot: págs. 4I5-426. 
En biomedicina los efectos físicos negativos (efectos adversos), tanto del producto en estudio como de la propia investigación, están previstos y se contempla compensación y reparación de tales daños (a través de los seguros), pero generalmente no se cuantifican los efectos psicológicos negativos. Por su parte, en una investigación social sobre el estilo de vida del mismo paciente, no se consideran los daños psicológicos (cognoscitivos, emocionales y sociales) desde la perspectiva de la complejidad, ni se confrontan sus propias deficiencias en caso de no poder hacerles frente.

En el experimento psicológico desarrollado por Philip Zimbardo en la prisión de Stanford sobre la influencia de tal ambiente extremo, en las conductas desarrolladas por sujetos dependiendo de sus roles sociales (cautivo o guardia) se concluyó que: a) la situación experimental lo que hace es poner condiciones para que se reproduzcan comportamientos de los que hemos sido previamente testigos, en lo que hemos sido entrenados, o que hemos sufrido: "en la escuela y en la vida familiar la gente aprende el significado del poder y de la obediencia a la autoridad y ha sido testigo de los roles de guardián y prisionero en diversas formas"31; y b) cuando a una persona la enfrentamos a situaciones sociales extremas, suele ganar la situación. Según el investigador: "Lo que hicimos [...] fue sencillamente colocar a la gente nueva dentro de una situación envenenada, y la situación ganó la partida"32.

\section{Eventuales riesgos en la Investigación en Ciencias Sociales}

Claramente el daño que se puede ocasionar, no es directo o letal como podría ocurrir en una investigación biomédica, si no, menos visible y explícito. A vía meramente ejemplar en un diseño experimental que desembocó en la imposición coercitiva del discurso de las emociones, la frase "¿cómo se siente?" era reiterada por Stanley Milgram en la Universidad de Yale para probar cuánto dolor infligiría un ciudadano común a otra persona simplemente porque se lo pedían para un experimento científico. Por sobre los imperativos morales de los sujetos participantes se impuso la férrea autoridad de lastimar a otros y, con los gritos de las víctimas sonando en los oídos de esos participantes, la autoridad subyugaba con mayor frecuencia. Por ello, el principal descubrimiento del experimento fue la extrema buena voluntad de los adultos de aceptar casi cualquier requerimiento ordenado por la autoridad 33 .

Es importante señalar que Milgram dice que lo esencial de la obediencia es que una persona llega a considerarse instrumento para realizar los deseos de otra, y por tanto deja de creerse responsable de sus propios actos. Una vez producida esta variación de perspectiva, se siguen todos los caracteres esenciales de la obediencia. El resultado más trascendental es que la persona se considera responsable ante la autoridad que la dirige, pero no del contenido de los actos que le

3I Evans, R. The Making of Social Psychology. Discussions whit Creative Contributors. NY: Gardner Press 1980, pág. 2.

32 ĺdem.

33 Cf. Milgram, S. The Perils of Obedience 1974, Harper's Magazine, December 1973, págs. 62-66, 75-77, [En línea] [citado el 10 de diciembre de 2016]. Disponible en: https://harpers.org/archive/1973//2/the-perils-of-obedience/ 
ordenan ejecutar. No desaparece la moralidad, sino que se toma el foco radicalmente diferente: la persona subordinada siente orgullo o vergüenza, según haya desempeñado bien o mal el cometido que le encargó la autoridad ${ }^{34}$.

Este experimento no consideró la voluntad de la persona, ni estuvo sujeto a normas éticas como la Declaración de Helsinki en su numeral 7. No se respetó a los seres humanos en cuanto a sus derechos individuales y a su salud. Así, se usaron consignas engañosas en la investigación, no era revelada la naturaleza real del experimento, no se consideró el resguardo de los participantes del estudio e incluso por ello, el consentimiento no fue en sentido estricto válido ${ }^{35}$.

Ciertos estudios observacionales (sean epidemiológicos, demográficos o conductuales), no suponen una intervención directa sobre el cuerpo, razón por la cual se consideran inocuos, aunque dicha consideración debiera ser revisada. Tales estudios utilizan instrumentos de medición como análisis del discurso, entrevista en profundidad, historia de vida, sondeo de opinión, test psicométrico, entre otros, que permiten por una parte recabar información sobre prácticas de: aborto, abuso infantil, cohabitación, consumo de sustancias psicoactivas, delincuencia infantil o juvenil, enfermedades mentales, fertilización artificialmente asistida, incesto, maltrato laboral (también conocido como mobbing laboral o acoso moral), orientación sexual, violencia doméstica, institucional o simbólica; pero simultáneamente obligan (sin coerción) a las personas contactadas a revelar datos personales y sensibles y a rememorar situaciones de alto impacto psíquico.

Asimismo, en ese tipo de investigación (especialmente en aquellas referidas a trastornos reproductivos, identidad de género, paternalismo, parentalismo y problemas como el aborto, la esterilidad, la muerte o el suicidio) se puede provocar que en el sujeto puedan sobrevenir recuerdos de situaciones íntimas, dolorosas o violentas, otras sociales y del entorno familiar, todo lo cual al revivenciarlo pudiere causarle revictimización angustia y depresión ${ }^{36}$.

Es claro entonces que no es posible evaluar la investigación en, con y para comunidades o personas (sean estas sujetos primarios o sujetos secundarios ${ }^{37}$ ), con los mismos parámetros que se hace para la investigación clínica y en general biomédica. En virtud de ello, "Sólo hay que pedir permiso a una persona cuando se le va a introducir un tubo, o se le va a sacar un trozo de piel, o se le va a dar un fármaco nuevo?" 38 y "¿Qué pasa cuando, como ocurre en las Ciencias Sociales, no nos introducimos

34 Milgram, S. Los peligros de la obediencia. En Obediencia a la autoridad. Un punto de Vista Experimental. Bilbao, Desclée de Brouwer, 1980.

35 Milgram, S. Some Conditions of Obedience and Disobedience to Authority. Human relations, 1965, I8(I), pág. 57. Milgram, S. Obedience to Authority; An Experimental View. London, Tavistock, 1974.

36 Leibovich de Duarte, A. "La dimensión ética en la investigación psicológica", Revista Investigaciones en Psicología. Buenos Aires, Facultad de Psicología-UBA, 2000: págs.4I-6I.

37 Oficina de Regulación en Salud de los Estados Unidos. The Code of Federal Regulations (CFR) 45-46.II6 (d) Definición de "sujeto secundario" a cualquier persona a la cual un/a investigador/a solicita información referente a su propia condición (física o psíquica) o a la de otra persona en estudio.

38 Michaud Chacón, P. "Introducción" en E. Lira Korinfeld (ed.) Bioética en Investigación en Ciencias Sociales. CONICYT-Gobierno de Chile, Santiago, 2008, pág. I 3. Los destacados en bastardilla nos pertenecen. 
en su cuerpo físico, sino en su mente, sus sentimientos, sus deseos, sus valores, su alma, su espíritu como se quiera llamar?"39.

En ocasiones como se señaló, puede tratarse de una investigación de apariencia netamente clínica pero que incluye temas especialmente sensibles, "especialmente aquellas que involucran infecciones de transmisión sexual (ITS-incluyendo el VIH), cáncer y algunas crónico-degenerativas, pueden contribuir a dañar a los participantes [...Por ello], "la responsabilidad de los investigadores y Comités de Ética en evitar daños potenciales es sumamente importante y debe estar en el centro de las reflexiones en la ética de toda investigación en salud'40.

La premisa que supone que los estudios sociales y/o comportamentales no implican riesgo, potencia y multiplica (por acción o por omisión) las instancias de angustia, de depresión o de trauma, habida cuenta que el problema subyacente en tales estudios, radica en la dificultad de realizar un efectivo balance entre riesgos y beneficios. Es menester entonces, resguardar aún más la confidencialidad, teniendo en cuenta la vulnerabilidad (permanente o transitoria) de las personas involucradas.

Toda interacción que involucre una intervención sobre los pensamientos, los sentimientos, las preferencias y/o los valores de las personas en estudio, podría resultar cruenta, intrusiva y de riesgo más que mínimo, produciendo perturbaciones y por eso se requiere evitarlas o disminuir el riesgo al máximo. Dado que "lo primero es no dañar" las potenciales lesiones deben ser controladas y evitadas desde el mismo momento en que se diseña un protocolo de investigación.

El ser humano es un conjunto de elementos biológicos, redes de conexiones, valores, reacciones y sentimientos, único e indivisible, que vive y responde ante la enfermedad de diferentes maneras. Muchas veces no se contempla apoyo médico ni compensación de daños. Si la investigación necesitó "penetrar" en la intimidad de la vida de la persona para responder al objetivo de acumulación de conocimientos, o sobre posibles políticas o programas de prevención o de atención de salud, es probable que tanto el investigador como el participante no hayan medido las consecuencias personales de las preguntas y que el consentimiento haya sido tácito, pero no por ello con menos riesgo.

El investigador debe aclarar que el aporte de la investigación depende de la veracidad de las respuestas, las cuales deben depender de la legitimidad al hacerlas. Al presentarse y aclarar sus objetivos, quien investiga debe también dejar establecido que el resguardo de la esfera privada e íntima de las personas (garantizando su anonimato y la confidencialidad de sus datos), serán preservadas a través de los medios establecidos para tal efecto. Esto durante toda la investigación y también en instancias posteriores, de divulgación y/o de publicación de la información obtenida. Pero, el eventual "blindaje" de la información, aun cuando sea acordado previamente, podría generar suspicacias en las personas estudiadas, por lo general, debido a experiencias previas de filtración de información.

39 Ídem.

40 Sánchez Pérez, H.; Benites Estupiñan, E.; Quiroz Malca, E. y Vergès de López, C. "Patrimonio indígena: ¿investigación o biopiratería? Responsabilidad ética" en P. Sorokin (coord.) Número Especial de Jurisprudencia Argentina sobre Derechos personalísimos y $\mathrm{VIH} /$ sida en el nuevo decenio. Buenos Aires: AbeledoPerrot, I (8), (20I I), pág. 73. 
Algunos recomiendan que el participante tenga acceso a sus respuestas y pueda corregirlas antes del análisis para la investigación, puesto que hay una gran distancia entre lo que se dice y lo que se escribe e interpreta. De igual manera la posibilidad de retiro sin sufrir perjuicio o sanción debiera estar contemplada. En realidad, con adaptaciones de contenido se podría concluir que los "principios éticos" de la investigación biomédica resultan aplicables a las investigaciones sociales en el campo de la salud, porque los daños son similares, aunque afecten diferentes componentes del ser humano.

Las diferencias pueden estar más que todo en la presentación de la información necesaria para un consentimiento autónomo, en el respeto de la dignidad de las personas, grupos sociales o étnicos y en la necesidad de prever, minimizar y eventualmente compensar posibles daños. Como lo han demostrado justamente las ciencias sociales (Weber, Ivan Illich), el auto-control no es suficiente para garantizar la protección de los participantes. Por ello los investigadores sociales al ser conscientes de los riesgos que se corren en los proyectos propuestos, debieran considerar como necesario que sus protocolos de investigación sean revisados por Comités de Ética y que a su vez éstos adquieran las competencias metodológicas indispensables en este nuevo escenario, esto es la hermenéutica bioética.

En un contexto de desarrollo disciplinario (de la bioética y de la ética de/en la investigación) en el cual los aportes técnicos emanados de profesionales provenientes de las ciencias sociales y humanas así como de las ciencias del comportamiento, pudieren ser resistidos o poco valorados, se requiere reflexionar interdisciplinaria, intersectorial e internacionalmente en torno de ciertas cuestiones que deben ser incluidas tanto en las agendas académicas, como en las prioridades de los formuladores de políticas públicas en y de investigación.

\section{A modo de epílogo}

Habida cuenta de que la inclusión de cientistas sociales en los Comités de Ética, abre nuevos horizontes sobre los métodos de investigación y perspectivas conceptuales propias, cabe destacar la importancia y la necesidad de remitir los protocolos de investigación en ciencias sociales (aun aquellos que a simple vista sean considerados "sin riesgo") a la evaluación de un Comité de Ética de la Investigación acreditado. Dichos Comités, con esta estructura, garantizarían la aplicación de la hermenéutica en la búsqueda de la comprensión de las dimensiones posibles de la investigación, considerando obviamente los contextos socioculturales donde se desarrollen, logrando así no solo la protección de las personas sino el resguardo de sus concepciones íntimas; ideas, pensamientos y tradiciones, entre otras.

Podría asumirse la urgente necesidad de regular jurídica y específicamente la investigación en ciencias sociales, humanas y del comportamiento, tanto durante el desarrollo en general del proyecto, como en todas las acciones conexas. La anonimización de los datos personales o colectivos y la disociación de los datos sensibles, si bien necesaria no es suficiente resguardo frente a casos únicos o de ocurrencia muy rara, por lo que la normativa que se expida al respecto debe considerar claramente los escenarios, modalidades y metodología empleada en la investigación social, humana 
y comportamental, incluyendo las fases correspondientes a publicación de resultados parciales o totales, positivos o negativos.

El fin último de toda investigación no consiste solo en "saber" sino en "saber para" intervenir, para prevenir, para comprender, para cambiar, para garantizar igualdad, equidad y justicia, para evitar dolor innecesario, para mejorar la vida, en sus condiciones, en su calidad y entre otros objetivos, el proyecto de vida y las esperanzas de las personas que de éstas participan.

La integridad psicofísica de las personas es mucho más que un simple requisito legal o un trámite administrativo, es "prima facie" un derecho humano en ejercicio. Ello implica, por parte de los Comités, el cumplimiento (que no siempre se da aun cuando las directivas de UNESCO en la materia así lo establecen) de criterios tales como: interdisciplinariedad, distribución por género, calificación en la materia, ausencia de conflictos de interés, presencia de expertos y no expertos y transparencia, entre otros.

Como premisa fundamental, el criterio ético que regula esta ciencia es el respeto al ser humano, a sus derechos inalienables, a su bien verdadero e integral, en definitiva, a la dignidad de la persona.

\section{Bibliografía}

Asociación Médica Mundial (AMM). Declaración de Helsinki, Principios éticos para las investigaciones médicas en seres humanos, 64a Asamblea General, Fortaleza, Brasil, 20 I3. [En línea] [Citado el 10 diciembre de 20I6]. Disponible en: http://www.wma.net/es/30publications/I Opolicies/b3/

Beecher, Henry K. The Powerful Placebo. 1955. Journal of the American Medical Association I59 (17):1602I 606. ISSN 0002-9955. doi: I0. I00 I/jama. 1955.02960340022006.

ClOM/OMS. Pautas éticas internacionales para la investigación biomédica en seres Humanos. Ginebra 2002. [En línea] [Citado el 10 diciembre de 2016]. Disponible en: http://www.cioms.ch/publications/guidelines/ pautas eticas internacionales.htm

Comisión Nacional para la protección de sujetos humanos de investigación biomédica y comportamental. El Informe Belmont. U.S.A. 1979. [En línea] [Citado el 10 diciembre de 2016]. Disponible en: http://www. bioeticayderecho.ub.edu/archivos/norm/InformeBelmont.pdf

Conferencia Internacional de Armonización (ICH) Normas de Buenas Prácticas Clínicas. (BPC) 1997-1998. [En línea] [Citado el 10 diciembre de 20 I6]. Disponible en: http://www.fda.gov/downloads/Drugs/.../Guidances/ ucm073|28.pdf

De Abajo Iglesias, F. Un caso histórico: los estudios de hepatitis de la escuela estatal de Willowbrook Investigación pediátrica clínica y traslacional en la era genómica. S/I, 20 I2. [En línea] [Citado el I0 de diciembre de 20 I6]. Disponible en: http://contenidos.institutoroche.es/pdf/20 I2/libro_pediatria/anexos_capt4.pdf

Evans, R. The Making of Social Psychology. Discussions whit Creative Contributors. NY: Gardner Press 1980, pág. 2 
Gracia, D. Bioética Clínica, Ed. El Búho, Bogotá, 1998, págs. 39.

Gracia D. Profesión médica, investigación y justicia sanitaria, Ed. El Búho, Bogotá, 1998, pág. 67.

Kant, I. Cimentación para la metafísica de las costumbres. C. M. Ramírez (trad.) Buenos Aires, Aguilar, 5a. Ed. 1978, págs. I | |- | 12.

Leibovich de Duarte, A. "La dimensión ética en la investigación psicológica", Revista Investigaciones en Psicología. Buenos Aires, Facultad de Psicología-UBA, 2000, págs. 4I-6I.

Mainetti, J. A. Bioética y Medicina Poshumanística. Editorial Quirón, La Plata. 2013, pág. 60.

Michaud Chacón, P. "Introducción" en E. Lira Korinfeld (ed.) Bioética en Investigación en Ciencias Sociales. CONICYT-Gobierno de Chile, Santiago, 2008, pág. 13. Los destacados en bastardilla nos pertenecen.

Milgram, S. Los peligros de la obediencia. En Obediencia a la autoridad. Un punto de Vista Experimental. Bilbao, Desclée de Brouwer, 1980.

Milgram, S., Obedience to Authority; An Experimental View. Harpercollins (ISBN 006131983 X). Editor London: Harper Collins. 1974.

Milgram, S. Some Conditions of Obedience and Disobedience to Authority. Human relations, 1965, I8(I), pág. 57.

Milgram, S. The Perils of Obedience 1974, Harper's Magazine, December 1973, págs. 62-66. 75-77, [En línea] [citado el 10 diciembre de 20 16]. Disponible en: https://harpers.org/archive/ /973/I2/the-perils-of-obedience/

Naciones Unidas, Declaración Universal de Derechos Humanos, Adoptada por la Asamblea General de las Naciones Unidas en su Resolución 217 A (III), el 10 de diciembre de 1948 en París, Francia. [En línea]. [Citado el 10 diciembre de 2016] Disponible en: http://www.un.org/es/documents/udhr/

Naciones Unidas, Pacto Internacional de los Derechos Civiles y Políticos, 1966, art. 17. [En línea] [Citado el 10 diciembre de 2016]. Disponible en: http://www.ohchr.org/SP/Professionallnterest/Pages/CCPR.aspx

Naciones Unidas, Pacto Internacional de los derechos Económicos, Sociales y Culturales, 1966. [En línea] [Citado el 10 diciembre de 2016]. Disponible en: http://www.ohchr.org/SP/Professionallnterest/Pages/CESCR.aspx

Oficina de Regulación en Salud de los Estados Unidos. The Code of Federal Regulations (CFR), págs. 45-46. I 16

Sánchez Pérez, H.; Benites Estupiñan, E.; Quiroz Malca, E. y Vergès de López, C. "Patrimonio indígena: investigación o biopiratería? Responsabilidad ética" en P. Sorokin (coord.) Número Especial de Jurisprudencia Argentina sobre Derechos personalísimos y VIH/sida en el nuevo decenio. Buenos Aires: Abeledo Perrot, I (8), (20I I), pag. 73.

Sorokin P., Valerio C. y Cecchetto S. "Poblaciones vulnerables e investigación biomédica: A propósito de un caso" en S. Bergel (coord.) Número especial de Bioética y Derechos Humanos de la Revista jurídica de Buenos Aires. UBA-LexisNexis-AbeledoPerrot. Buenos Aires: 2006-2007, págs. 4I 5-426.

Sorokin P, Sotomayor Saavedra M. A, Vergès de López C, Actis A, Gubert I. C, Benites Estupiñan E, López Dávila L.M, Quiroz Malca E, Scrigni A. y Rueda Castro, L. "Perspectivas bioéticas en torno de la necesidad de regular 
la investigación en ciencias sociales y del comportamiento". $45^{\circ}$ Congreso de la Sociedad Internacional de Historia de la Medicina. Facultad de Medicina-UBA, Buenos Aires, Argentina: 06/09/2016.

Ulin P., Robinson E. y Tolley E. Investigación aplicada en salud pública. Métodos cualitativos. OPS / OMS, Washington D.C. 2006, 286 págs. [Publicación Científica y Técnica No. 614]

UNESCO. Ethical Guidelines for International Comparative Social Science Research in the framework of MOST, Artículo 10. 2016. [En línea] [citado el 10 diciembre de 2016]. Disponible en: http://www.unesco.org/most/ ethical.htm

Winkler, M. I.; Alvear, K.; Olivares, B. y Pasmanik, D. Querer No Basta: Deberes Éticos en la Práctica, Formación e Investigación en Psicología Comunitaria. Psykhe. 2012, vol.21, n. I, págs. I I5-129. [En línea] [citado el 10 diciembre de 20 I 6]. Disponible en: http://dx.doi.org/I 0.4067/S07 | 8-222820 I 2000 I 00008 